\title{
EP-35
}

\section{Comparison of short-term and long-term survival groups and prognostic factors in patients with liver metastasis of colorectal cancer}

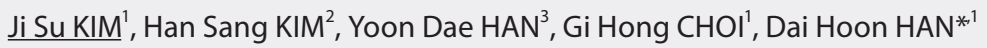 \\ 'Department of Hepatobiliary and Pancreatic Surgery, Severance Hospital, Yonsei University College of Medicine, Seoul, Korea \\ ${ }^{2}$ Division of Medical Oncology, Department of Internal Medicine, Severance Hospital, Yonsei University College of Medicine, Seoul, Korea \\ ${ }^{3}$ Division of Colorectal Surgery, Severance Hospital, Yonsei University College of Medicine, Seoul, Korea
}

Introduction: There was no report for comparing long-term survival group with short-term survival group in patients who underwent liver resection for colorectal cancer with liver metastasis. Here, we compared the difference between the short-term and longterm survivors and analyzed the factors that influence the prognosis.

Methods: From January 2016 to December 2018, liver resections were performed for 478 patients of liver metastasis at the Yonsei University Severance Hospital. Except 111 patients due to lack of data, total 367 patients were analyzed retrospectively. Patients were divided into two groups: those who survived less than 24 months (short-term survival group, $\mathrm{n}=87$ ) and those who survived more than 24 months (long-term survival group, $\mathrm{n}=280$ ).

Results: In the short-term survival group, there were more patients with tumor size of $2 \mathrm{~cm}$ or more than the long-term survival group $(p=0.017)$. And the short-term survival group had more patients with margin positive than the long-term survival group $(p<$ 0.05). The long-term survival group had more patients with single lesion than the short-term survival group $(p=0.028)$. In cox regression analysis, tumor size, tumor number, and resection margin were the prognostic factors effect survival.

Conclusions: For long-term survival of patients with liver metastasis of colorectal cancer, when deciding whether to undergo liver resection, whether a tumor smaller than $2 \mathrm{~cm}$ or a single lesion or curative resection is possible should be considered. 